\title{
Lyme disease prevention and control - the way forward
}

\author{
Beard $\mathrm{CB}^{1 *}$ \\ ${ }^{1}$ Division of Vector-Borne Diseases, U.S. Centers for Disease Control and Prevention (CDC), \\ Fort Collins, CO \\ *Corresponding author: cbeard@cdc.gov
}

\section{Introduction}

Lyme borreliosis is a tick-borne illness caused by the spirochete Borrelia burgdorferi, harbored primarily by small rodents, and transmitted by ticks in the genus Ixodes. It was first recognized clinically in the 1970s, in a cluster of juvenile arthritis cases in Connecticut (1). Over the last twenty five years, Lyme disease has been increasing steadily in the United States, both in case numbers and in geographic distribution. As noted in the other articles in this issue, Lyme disease has started to show a similar pattern in Canada. In the United States, there were over 30,000 cases reported to the Centers for Disease Control and Prevention (CDC) in 2012, making it the 7th most common reportable condition. The increasing numbers of cases is thought to have resulted from expanding deer populations and suburban growth that have led to abundant reservoir hosts, more ticks, and greater opportunities for exposure in humans (2). It is important to note that the observed trends for Lyme disease emergence are similar for several other tick-borne diseases that share similar ecologies, including anaplasmosis, babesiosis, ehrlichiciosis, and Powassan disease.

Symptoms of Lyme disease range from an erythema migrans rash, early in the course of infection, to neuritis (e.g., facial palsy), carditis, and arthritis in later, disseminated stages of illness. Prompt treatment with 2-4 weeks of oral doxycycline results in symptomatic cure of the great majority of patients. Nevertheless, a subset of patients, especially those diagnosed and treated in later stages of illness, may have persistent fatigue, muscle aches, short-term memory problems, and other nonspecific symptoms. Consequently, one of the highest priority research needs in the field of Lyme disease is to elucidate the specific cause or causes of symptoms in these patients and to determine the safest and most effective treatment options. This question is the focus of several current or recently published research studies in humans and non-human primates that are evaluating treatment response, clinical outcome, and the possibility of spirochete persistence following treatment (3-5).

Another very high priority research need is for improved diagnostics. The currently validated diagnostic tests in common use for Lyme disease are all serologic tests that rely on a detectable antibody response. Consequently, these tests have limited value early in infection or in patients who have had prior infection, depending on how long ago the infection occurred. Some of the more promising research that is ongoing in this area focuses on the identification of direct diagnostic targets. These may either be nucleic acids or low molecular weight cellular metabolites that are indicators of active infection or a specific host-mediated response to infection.

One last highly critical research need worth noting is for the development and evaluation of safe and effective prevention and control tools and methods. This topic will be discussed further below.

In addition to these research needs, other critical needs must be addressed in order to establish and maintain an effective public health response. These include 1.) an accurate understanding of disease distribution and risk; 2 .) an awareness of the disease among the public at risk and knowledge about how to protect themselves; 3 .) informed healthcare providers who can recognize the disease and provide early and accurate diagnosis and treatment; 4.) validated prevention and control practices; and 5.) effective multi-level collaboration toward the goal of prevention. The remaining sections of this paper briefly discuss each of these topics. 


\section{Future Efforts}

\section{Disease distribution and risk}

The starting point for an effective public health response to Lyme disease is to maintain accurate information on disease distribution and the specific locations and areas of risk. This is required to track disease trends and project future emergence, and is critical for targeting prevention education and resources for control. This is particularly true for emerging diseases like Lyme disease where the disease is expanding into areas where it did not previously exist and may not be recognized. It is also important to understand local disease ecology and epidemiology so that interventions can be targeted effectively to particular locations where infection risk is greatest.

\section{Public awareness}

Once the specific region or regions of enzootic activity and the specific epidemiologic risks have been identified, there is a need to raise awareness in these at-risk communities. Some of the methods available include the use of trail signs, public service announcements, and prevention information targeted to specific high-risk groups. Resources are increasingly available, both in Canada (6) and in the United States (7). Targeting prevention messages to areas where the disease is newly emerging will make newly at risk populations more aware of the problem and of what can be done about it. These groups may also be more eager to engage.

\section{Healthcare provider education}

Healthcare provider education is highly important in these areas of emerging risk. Despite the controversy that surrounds treatment of patients with persistent symptoms associated with Lyme disease, a common point of agreement is that early and accurate diagnosis and treatment (also referred to as secondary prevention) is the most effective way to reduce serious clinical outcomes. A recent report of Lyme carditis and sudden cardiac death emphasizes the need for vigilance in Lyme endemic areas to ensure prompt recognition and early, appropriate therapy (8). Healthcare providers also play an important role in prevention education by informing their patients about the ways to reduce their exposure to vector ticks. Several courses are now available for continuing medical education credit as well as other useful information (9-10).

\section{Prevention and control practices}

The most useful prevention tool for Lyme borreliosis would be a safe and effective human vaccine that has been adequately evaluated in both children and adults. No such vaccine is available at this time. Consequently, Lyme disease prevention recommendations currently include activities aimed at reducing exposure to infected ticks. These methods include such practices as area pesticide applications, landscape management, deer management, applying repellants or toxicants to skin or clothing, tick checks, showering after being in tick habitat, and host-targeted interventions (11). Some examples of the latter include reservoir-targeted vaccines, 4-poster deer devices, and bait boxes that contain acaricides that kill ticks on rodent hosts. In general, while these methods can reduce the numbers of ticks, scant evidence exists that their use actually reduces human illness. The human Lyme disease vaccine LYMErix ${ }^{\mathrm{TM}}$ was the only intervention that was ever demonstrated in large community trials to reduce human illness (12). In the absence of a human vaccine, the most effective efforts to reduce exposure to infected ticks will probably require a combination of personal protective measures and coordinated local activities aimed at reducing tick densities in places where people are most likely exposed. In evaluating these measures, however, true prevention outcomes should be assessed. Many studies have looked at the reduction in entomological risk (i.e. the reduction in abundance of infected nymphal ticks), but few measure the actual reduction in physician-diagnosed disease or a suitable proxy such as the numbers of ticks found on people in treated versus untreated groups (13). 


\section{Multi-level collaboration}

The fifth and final critical need is for multi-level collaboration in the development and implementation of disease prevention efforts. Effective control begins at the local level, in the backyards, neighborhoods, parks, and green spaces where infected ticks are encountered. The best solutions for prevention are likely local solutions that account for variation in local disease ecology, the corresponding unique patterns of risk, and population preferences. Public health agencies at federal, state, and provincial levels, however, can more easily conduct or fund evaluation studies and can also draw from the experience of multiple communities across broad geographic areas. Having identified and validated the best prevention practices, they can then make this information available in the form of evidence-based guidelines and recommendations. Through their influence, they can stimulate communication and collaboration among the key public health partners, such as local health departments and community groups, including patient advocacy groups. People are likely to encounter infected ticks in multiple locations, including their own yards, their neighbor's yard, and public areas. For this reason, effective collaboration and coordination among key prevention partners at multiple levels will be essential in achieving lasting control. The Public Health Agency of Canada has recently launched a national Action Plan on Lyme Disease that will inform these efforts (14).

\section{Conclusion}

Lyme and other tick-borne diseases are on the increase in Canada and the U.S. There are a number of very important needs that are being addressed through current research, including improved diagnostic tests, an understanding of the cause of persistent symptoms and the corresponding best treatment options, and a safe and effective vaccine. An effective public health response depends upon an accurate understanding of the problem, knowledge and awareness among both healthcare providers and the population at risk, the availability of effective prevention and control methods that can reduce human illness, and the development and coordination of a national action plan that is applied locally, according to unique epidemiologic and ecological features of disease risk.

\section{References}

(1) Steere AC, Malawista SE, Snydman DR, Shope RE, Andiman WA, Ross MR, Steel FM. Lyme arthritis - An epidemic of oligoarticular arthritis in children and adults in three Connecticut communities. Arthritis and Rheumatism. 1977; 20(20):7-17.

(2) Steere AC, Coburn J, Glickstein L. The emergence of Lyme disease. J Clin Invest. 2004; 113(8):1093-1101.

(3) Klempner MS, Baker PJ, Shapiro ED, Marques A, Dattwyler RJ, Halperin JJ, Wormser GP. Treatment trials for post-Lyme disease symptoms revisited. The American Journal of Medicine (2013) 126, 665-669.

(4) Embers ME, Barthold SW, Borda JT, Bowers L, Doyle L, Hodzic E, Jacobs MB, Hasenkampf, NR, Martin DS, Narasimhan S, Phillippi-Falkenstein KM, Purcell JE, Ratterree MS, Philipp MT. Persistence of Borrelia burgdorferi in Rhesus Macaques following Antibiotic Treatment of Disseminated Infection. PLoS ONE 7(1): e29914. DOI:10.1371/journal.pone.0029914.

(5) Marques A, Telford SR, Turk S-P, Chung E, Williams C, Dardick K, Krause PJ, Brandeburg C, Crowder CD, Carolan HE, Eshoo MW, Shaw PA, Hu LT. Xenodiagnosis to detect Borrelia burgdorferi infection: A first-inhuman study. Clin Infec Dis. 2014. DOI: 10.1093/cid/cit939

(6) Public Health Agency of Canada. Lyme Disease Frequently Asked Questions. http://www.phac-aspc.gc.ca/id-mi/lyme-fs-eng.php.

(7) CDC. Lyme Disease Prevention Tool Kit. www.cdc.gov/lyme/toolkit.

(8) CDC. Three Sudden Cardiac Deaths Associated with Lyme Carditis - United States, November 2012-July 2013. Morbidity and Mortality Weekly Report. 2013; 62(49):993-96.

(9) CDC. Lyme Disease Resources for Clinicians. www.cdc.gov/lyme/healthcare/clinicians.html. 
(10) Public Health Agency of Canada. Lyme disease and other tick-borne diseases: Information for healthcare professionals. http://www.phac-aspc.gc.ca/id-mi/tickinfo-eng.php.

(11) Piesman J, Eisen L. Prevention of Tick-Borne Diseases. Annu Rev Entomol. 2008; 53:323-43

(12) Poland GA, Jacobson RM. The prevention of Lyme disease with vaccine. Vaccine. 2001; 19:2303-08.

(13) Connally NP, Durante AJ, Yousey-Hindes KM, Meek JI, Nelson RS, Heimer R. Peridomestic Lyme Disease Prevention: Results of a Population-Based Case-Control Study. Am J Prev Med. 2009; 37:201-06.

(14) Harymann M, Ogden N, Lindsay R, Lawless V, Dielgat M, Sternthal S. Public Health Agency of Canada. Summary of the Public Health Agency of Canada's Action Plan on Lyme Disease. CCDR 2014, Vol 40-5.

\section{Acknowledgments}

I would like to thank Drs. Paul Mead and Lyle Petersen and Ms. Anna Perea for feedback and constructive criticism on the contents of this paper. The findings and conclusions in this report have not been formally disseminated by the Centers for Disease Control and Prevention and should not be construed to represent any agency determination or policy.

\section{Conflicts of interest}

The author has no conflicts of interest to report. 